\title{
DOES TOPICAL FLURBIPROFEN AFFECT THE PUPILLARY RESPONSE TO ACETYLCHOLINE?
}

\author{
H. JACKSON ${ }^{\prime}$, C. K. PATEL ${ }^{2}$, M. WESTCOTT ${ }^{3}$, G. M. THOMPSON ${ }^{\prime}$ and B. M. MATHALONE ${ }^{3}$ \\ London, Oxford and Kingston-upon-Thames
}

\begin{abstract}
SUMMARY
Pre-operative topical non-steroidal anti-inflammatory drugs (NSAIDs) are known to be effective in maintaining pupillary dilation during cataract surgery. However, previous studies have suggested that pre-treatment with these agents interferes with the miosis produced by intraoperative acetylcholine injection. This study was designed to determine whether the pupillary response to acetylcholine injection is reduced following pre-operative topical flurbiprofen $0.03 \%$. Pupil diameters were measured with a millimetre rule, and the measurements verified using computerised image analysis of digitised video images. Eyes treated with flurbiprofen were compared with controls. There was no significant difference in pupillary diameter before corneal incision, but following aspiration of soft lens matter and insertion of intraocular lens the pupil was significantly larger in the flurbiprofen group $(p=0.002)$. At 1.0, 2.5 and $5.0 \mathrm{~min}$ utes following injection of acetylcholine no significant difference in the degree of miosis was detected. This study confirms that topical flurbiprofen maintains dilation during cataract surgery, and does not find any evidence to suggest that intra-operative response to acetylcholine is reduced.
\end{abstract}

With modern techniques in cataract surgery such as capsulorrhexis and phacoemulsification, it is increasingly important for the surgeon to have the benefit of maximum pupillary dilation. Intra-operative miosis due to iris manipulation or inadequate pre-operative dilation can lead to technical difficulties during the operation. Pre-operative topical non-steroidal anti-inflammatory agents such as flurbiprofen, a potent prostaglandin inhibitor, are known to be effective in the maintenance of pupillary dilation during cataract surgery. ${ }^{1.2}$

Many surgeons inject a cholinergic agonist such as acetylcholine at the end of surgery or following insertion

From: 'Department of Ophthalmology, St George's Hospital, London: ${ }^{2}$ Nuffield Laboratory of Ophthalmology, University of Ox ford. Oxford; "The Royal Eye Unit, Kingston-upon-Thames, Surrey, UK.

Correspondence to: $\mathrm{Mr}$ C. K. Patel, Nuffield Laboratory of Ophthalmology, University of Oxiord, Walton Street, Oxford OX2 $6 \mathrm{AW}, \mathrm{UK}$.

Eye (1994) 8, 329-331 C 1994 Royal College of Ophthalmologists of an intraocular lens (IOL), in order to avoid complications such as iris capture and decentration of the IOL. Miosis becomes even more important if there has been vitreous loss, to avoid vitreous being left behind in the anterior chamber, and possibly to aid in the insertion of an anterior chamber IOL.

It is possible that the intra-operative response to acetylcholine injection may be reduced following pre-operative non-steroidal anti-inflammatory agents. Studies using rabbits have yielded conflicting data. ${ }^{3-5}$ A human study has shown that eyes pre-treated with flurbiprofen before cataract surgery had a significantly larger pupillary diameter 5 minutes after acetylcholine injection, although there was no significant difference if the change in pupil size was analysed. The number of patients involved in this study was, however, small. ${ }^{6}$

\section{PATIENTS AND METHODS}

A randomised, double-masked prospective study of the effect of pre-operative flurbiprofen on the pupil size during cataract surgery was performed. One hundred and eighteen patients undergoing routine extracapsular cataract surgery were entered consecutively into the study. Those who had undergone previous surgery, glaucoma therapy or laser treatment were excluded, as were patients taking anticholinergic drugs or with a history of herpes simplex keratitis or bleeding disorders. Patients taking non-steroidal anti-inflammatory drugs were not excluded, but were noted and examined during analysis of the results, as were diabetic patients. Approval from the hospital ethics committee was obtained, as was informed written consent from patients enrolled into the study.

The patients were randomly assigned to two groups. One group received flurbiprofen sodium $0.03 \%$ and the other hypromellose $0.3 \%$ from the nursing staff. One drop was given every 30 minutes starting 2 hours before surgery for a total of four doses. All patients also received cylcopentolate $1 \%$ and phenylephrine $10 \%$ every 15 minutes starting 1 hour before surgery.

Surgery was performed by three surgeons (G.M.T., H.J. 
Table I. Pupil diameter and change in pupil diameter

\begin{tabular}{|c|c|c|c|}
\hline & Flurbiprofen & Control & $p$ value \\
\hline \multicolumn{4}{|l|}{ Pupil diameter } \\
\hline Mean before corneal incision & $7.91( \pm 0.84)$ & $7.71( \pm 1.0)$ & 0.26 \\
\hline Mean after IOL insertion & $6.17( \pm 1.10)$ & $5.54( \pm 1.08)$ & 0.002 \\
\hline Mean 1 min after acetylcholine & $5.07( \pm 1.29)$ & $4.66( \pm 1.02)$ & 0.06 \\
\hline Mean $2.5 \mathrm{~min}$ after acetylcholine & $4.75( \pm 1.23)$ & $4.37( \pm 0.98)$ & 0.06 \\
\hline Mean 5 min after acetylcholine & $4.54( \pm 1.25)$ & $4.26( \pm 1.06)$ & 0.24 \\
\hline \multicolumn{4}{|l|}{ Change in pupil diameter } \\
\hline Mean change before corneal section to after IOL insertion & $1.73( \pm 0.99)$ & $2.19( \pm 1.15)$ & 0.02 \\
\hline Mean change after IOL insertion to $1 \mathrm{~min}$ after acetylcholine & $1.11( \pm 0.64)$ & $0.88( \pm 0.60)$ & 0.054 \\
\hline Mean change after IOL insertion to $2.5 \mathrm{~min}$ after acetylcholine & $1.43( \pm 0.73)$ & $1.16( \pm 0.71)$ & 0.22 \\
\hline Mean change after IOL insertion to $5 \mathrm{~min}$ after acetylcholine & $1.70( \pm 0.76)$ & $1.8( \pm 0.82)$ & 0.58 \\
\hline
\end{tabular}

Values are the mean, in millimetres, $\pm \mathrm{SD}$.

and C.K.P.) using a standard extracapsular technique. Viscoelastic was used before the capsulotomy and again before insertion of the IOL. The viscoelastic was removed before injection of acetylcholine $0.5 \mathrm{ml}$ (1:100). Balanced salt solution was used for irrigating the anterior chamber, and all operations were recorded on video.

Pupillary diameter was recorded before corneal incision, after expression of the nucleus, after insertion of the IOL and removal of the viscoelastic, and then at 1.0, 2.5 and 5.0 minutes after acetylcholine injection. The measurements at 1.0 and 2.5 minutes were performed on all patients and the 5.0 minutes measurement was performed on 51 patients. A millimetre rule was used for measurements, which were accurately verified using computerised image analysis of digitised video images. A two sample (two-tailed) Student's $t$-test was used to compare pupil diameters between the two groups. A $p$ value of less than 0.05 was considered significant.

\section{RESULTS}

Ten patients were excluded due to poor or incomplete video images. Five were from the flurbiprofen group and 5 from the control group. Six patients were excluded due to intra-operative problems such as vitreous loss or recurrent iris prolapse. Two were from the flurbiprofen group and 4 from the control group. The numbers are too small to draw any conclusions from this.

Fifty-seven patients were treated with flurbiprofen and 45 acted as controls. There were 16 males and 41 females with a mean age ( $\pm \mathrm{SD}$ ) of 78.7 ( \pm 9.5 ) years in the flurbiprofen group, and 12 males and 33 females with a mean age of $78.8( \pm 7.8)$ years in the control group. Clinical variables that could affect pupil response to mydriatics were evenly distributed between groups as follows (flurbiprofen versus control): the racial mix was 51 vs 41 Caucasian, 4 vs 4 Asian, 2 vs 0 Afro-Caribbean; 9 vs 7 patients were diabetic, 6 vs 5 patients were using oral non-steroidal anti-inflammatory drugs and 2 vs 2 patients were on oral steroids. Table I summarises the results. There was no difference in mean pupillary diameter between the groups before corneal incision and after lens expression. However, following irrigation/aspiration, insertion of the IOL and removal of viscoelastic, the pupil diameter was sig- nificantly larger in the flurbiprofen group than in the control group.

Following injection of acetylcholine $0.5 \mathrm{ml}$, there was no significant difference in the absolute pupil size or change in pupil size at 1.0,2.5 and 5.0 minutes. If patients with diabetes mellitus or those taking oral NSAIDs were excluded, the statistical significance of the results was unchanged.

\section{DISCUSSION}

Our study shows that the mean pupil diameter is significantly larger following insertion of the IOL in eyes treated with flurbiprofen, confirming the findings of previous reports. 1.2

Flurbiprofen is believed to maintain mydriasis by reducing production of prostaglandins via the inhibition of fatty acid cyclo-oxygenase, an enzyme essential in the biosynthesis of prostaglandins. ${ }^{7.8}$ The role of prostaglandins in producing miosis secondary to surgical trauma is complex; cyclo-oxygenase is also necessary in the production of substances such as thromboxanes and prostacyclin, ${ }^{8}$ and these may also have a role. Prostaglandins have been shown to be primary mediators of many ocular inflammatory mediators, but substance $\mathrm{P}$ may also play a significant part," as may other neuropeptides. ${ }^{8}$ Previous animal studies have suggested that the miotic response to acetylcholine or carbachol may be reduced following pretreatment with a NSAID. Ozog et al. ${ }^{3}$ reported a reduced miotic response to anterior chamber irrigation with acetylcholine in albino rabbits pre-treated with flurbiprofen; however, Zimm et al..$^{+}$found no significant difference when they performed a similar experiment on pigmented rabbits. These studies differed from ours in one important way, namely that there was no cataract surgery with the concomitant iris manipulation and release of prostaglandins. The smaller study performed on humans by Holmes et al. $^{6}$ reported a larger pupil size following acetylcholine injection in the patients pre-treated with flurbiprofen, but when they analysed the change they found no significant difference. In the much larger study reported here we also found that the pupil size was larger at 1.0, 2.5, 5.0 minutes after acetylcholine, but in this study the difference was not statistically significant. Our 
findings also agreed with those of Holmes et al. in that if the change in pupillary diameter following acetylcholine injection was examined there was no significant difference (Table I); indeed the change in pupil size was actually greater in the flurbiprofen group, although this could be explained in part by the fact that the initial pupil size for this measurement (after IOL insertion: Table I) was greater in this group.

Acetylcholine is a direct cholinergic agonist acting at the level of the motor end plates. It acts within seconds, and its duration has been shown to be 20 minutes or less. ${ }^{+}$ There is no theoretical basis for an inhibition of the action of acetylcholine by a prostaglandin inhibitor such as flurbiprofen. The study reported here would seem to confirm this, as no significant difference in the pupillary size after acetylcholine was found between patients treated with flurbiprofen and controls.

It is concluded that flurbiprofen $0.03 \%$ aids in the maintenance of mydriasis during cataract surgery. Previous suggestions that flurbiprofen interferes with the miotic response to acetylcholine were not confirmed by this study.

Key words: Acetylcholine, Flurbiprofen, Image analysis, Surgical miosis.

\section{REFERENCES}

1. Drews RC, Katsev DA. Ocufen and pupillary dilation during cataract surgery. J Cataract Refract Surg 1989;15:445-8.

2. Heinrichs DA, Leith AB. Effect of flurbiprofen on the maintenance of pupillary dilation during cataract surgery. Can $\mathbf{J}$ Ophthalmol 1990;25:239-42.

3. Ozog MF, Zimm JL, Park R. Evaluation of flurbiprofenexposed irides to acetylcholine anterior chamber irrigation. $\mathbf{J}$ Cataract Refract Surg 1990;16:226-9.

4. Zimm JL, Holmes JM, Anderson C, Evans LS. Effect of topical suprofen and flurbiprofen on the miosis produced by anterior chamber irrigation with cholinergic agonists. J Cataract Refract Surg 1991;17:790-3.

5. Yee RW, Kocurek D, Hing-Sing Y, Smith G, Lam C. Effects of flurbiprofen and suprofen on the miotic activity of acetylcholine and carbachol. Ophthalmol Surg 1992;23:528-32.

6. Holmes JM, Jay WM. The effect of preoperative flurbiprofen on miosis produced by acetylcholine during cataract surgery. Am J Ophthalmol 1991;111:735-8.

7. Stark WJ, Fagadau WR, Stewart RH, et al. Reduction of pupillary constriction during cataract surgery using suprofen. Arch Ophthalmol 1986; 104:364.

8. Camras CB, Miranda OC. The putative role of prostaglandins in surgical miosis. Prog Clin Biol Res 1989;312:197-210.

9. Bito LZ. Surgical miosis. Have we been misled by a bunch of rabbits? Ophthalmology 1990;97:1-2. 